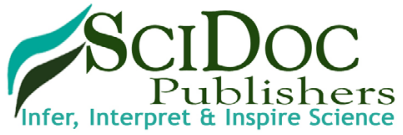

\section{The Development and Current Practices for Enhanced Recovery Following Colorectal Resection in The United Kingdom}

Brief Report

Hariharan V

Department of Colorectal Surgery, Whipps Cross University Hospital, London.

\title{
Abstract
}

Enhanced recovery following colorectal resection is a set of interventions which, when combined, lead to early return of gut function, fewer complications and a shorter length of stay. It divides the patient's journey into stages and ensures the surgical stress inflicted onto the patient is minimised and normalisation can occur as soon as possible. There has been much research into this new and advancing field with several meta-analyses confirming its benefit.

Keywords: Laparoscopic; Colorectal; ERAS; ERP; Enhanced Recovery Program; Enhanced Recovery After Surgery; Bowel Cancer; Nutrition; Analgesia.

\section{Introduction}

Laparoscopic colorectal surgery has led the way to the establishment of the enhanced recovery programme (ERP) or "Fast track" pathway as it is sometimes known. Indeed its demonstrated success in colorectal surgery has allowed it to be adopted into a wide range of allied surgical specialities [1].

From its conception in early 2000, Henrik Kehlet coined the phrase 'enhanced recovery programme' and published his remarkable findings of reduced operative morbidity and length of stay [2]. The combination of the ERP and laparoscopic surgery is synergistic and to this day, the vast majority of colorectal units in the UK follow an abbreviated form of the original ERP.

\section{What is the ERP?}

Traditional hospital stay of 10 - 14 days following major bowel resection had been accepted as normal practice up until fairly recently. However, in 2000, Basse and Kehlet [3] described a clinical pathway to accelerate recovery after colonic resection which dramatically cut down length of stay. Their study described a median stay of 2 days with a readmission rate of $15 \%$.

After study and development, a consensus on what the ERP core protocol contained was formed by Fearon et al. in 2005 [4].
The principles of care are based on:

\section{Pre-operative interventions \\ 2. Peri-operative interventions 3. Post-operative Care}

Enhanced recovery following colorectal surgery aims to minimise the stress response on the body and return gut function as rapidly as possible. It can be thought of as a combination of elements which together reduce the morbidity and length of stay post colorectal resection. Many of the interventions aim to address postoperative ileus which is a major hurdle to overcome for recovery following colorectal surgery. In addition to minimising the stress response to surgery, the aim is to speed recovery and return to normal function, thus minimising complications and improving overall outcomes. Over the last decade, there has been an increasing consensus as to what denotes an enhanced recovery programme. It is important to stress that a multidisciplinary approach is mandatory for ERAS to function. This must incorporate willingness from hospital staff as well as the patient and the relatives. A relatively recent role is that of the "Enhanced Recovery Nurse Specialists" or indeed "champions". They are a central figure who coordinates the multi-modal aspects of care including input from allied specialities such as the ward nurses, pain team, dieticians and physiotherapists. They work by enabling the patient's progress and views, and removing barriers to a successful recovery.

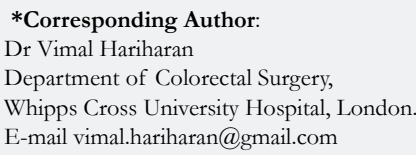

Citation: Hariharan V (2016) The Development and Current Practices for Enhanced Recovery Following Colorectal Resection in the United Kingdom . Int J Surg Res. 3(2), 43-46. doi: http://dx.doi.org/10.19070/2379-156X-160009

Copyright: Hariharan $\mathbf{V}^{\circ}$ 2016. This is an open-access article distributed under the terms of the Creative Commons Attribution License, which permits unrestricted use, distribution and reproduction in any medium, provided the original author and source are credited. 


\section{Pre-operative interventions}

Major surgery produces a stress on the body which leads to increased metabolic demand and nitrogen consumption. Typically the patient will be in a catabolic state for the first few days post operatively and will lose lean muscle mass rapidly as the body tries to replenish its nitrogen stores.

The introduction of "carbohydrate loading" 2 hours preoperatively decreases post-operative insulin resistance and negative nitrogen balance, thus reducing post-operative complications. Several RCTS have shown this benefit along with the importance of maintaining post-operative oral nutrition as well [5]. However this is not universally supported.

Patients find bowel preparation difficult to tolerate and troublesome to manage. Along with the dehydration and potential renal sequelae of the mass osmotic loss of fluids, patients themselves find the discomfort and disturbance the night prior to their surgery impacts on their physical and emotional status. It stands to reason that "a good night's sleep" before major surgery should be sought if at all possible. Multiple systematic reviews and meta-analyses have assessed the role of bowel preparation and concluded that mechanical bowel prep does not reduce anastomotic leakage and may increase wound infection rates [6].

Most surgeons now use bowel preparation for patients undergoing TME (as a column of faeces between the stoma and defunctioned anastomosis could theoretically increase the chance of anastomotic dehiscence). For left sided or high rectal anastomoses we would employ a simple enema at the time of surgery.

\section{Key Points for Clinical Practice}

Pre-operative care incorporates optimisation of the patient, ensuring adequate hydration and minimisation of starvation.

- Admission on the day of surgery

- Judicious use of bowel preparation

- Carbohydrate pre-loading

- Careful counselling of the patient

\section{Peri-operative interventions}

Perioperative care starts with minimising the insult of surgery. With the advent and progress of laparoscopic surgery, wounds are now much smaller; access to parts of the abdomen that previously would have needed larger incisions can be accomplished with incisions that do not need closing. There is less tissue manipulation, retraction and stress [7]. This facilitates reduced anaesthetic and analgesic requirements and in turn leads to reduced bed rest and an earlier return to normal function.

The laparoscopic approach is only a facet of the ethos enhanced recovery. Certainly it lends itself to the programme but in no way does an operation not performed laparascopically (wholly or partly) exclude itself from using the tools of enhanced recovery. As discussed earlier, enhanced recovery is making its way into all the surgical specialities and is not confined to colorectal resection solely [1].

The perioperative preparation starts in the anaesthetic room. The anaesthetist plans the anaesthetic in close conjunction with the surgeon. Consideration is given as to the type of agents used; pre-medication is now avoided; agents with long half-lives and opiates are minimised. The patient is minimally starved and is relatively well hydrated. Peri-operative analgesia is planned; mid thoracic epidural or transversus abdominus plane (TAP) blocks and patient controlled analgesia (PCA) are selected depending on the unit's preference. There are issues however with epidural anaesthesia. Hubner et al. randomised 128 patients to epidural vs PCA and found the epidural arm to slow down recovery and increases the need for vasopressor support [8]. Patient pain scores on day one were unchanged. Combined with the accepted $10 \%$ failure rate of epidurals, they go on to strongly state that this mode of anaesthesia should not be recommended. Total intravenous anaesthesia (TIVA) is becoming more common place, and agents such as Remifentanil have ultra-short half-lives of 4 minutes leading to a "fast on, fast off" effect. Other methodologies exist such as single shot spinal anaesthesia, infusion catheters and peripheral antagonists but the principle remains the same: a comfortable patient post-operatively who is able to return to function rapidly.

When clinically appropriate, a nasogastric drainand post operative drains should be avoided to aid early patient mobilisation. No benefit for routine anastomotic drainage has been found by a recent Cochrane review [9]. Intra operative use of non invasive monitoring should be used for goal directed fluid replacement. Central venous and arterial line catheters are no longer standard for ASA grade I and II patients.

Fluid balance is a topic of much controversy with many contradictory findings from trials. However the evidence is clear that gut function and tissue healing, morbidity and hospital stay are adversely affected by the overuse on intravenous fluid therapy [10]. This, along with Brandstrup's work [11], show a clear survival benefit to those patients who are fluid restricted as opposed to those that receive large sodium and volume loads. It was shown that a patient who received $<2$ litres and $77 \mathrm{mmol}$ of sodium per day showed improved gastric emptying and reduced complications. It is not clear as to the cause of this effect but we must remember that during surgical stress, the body retains salt and water via anti-diuretic hormone $(\mathrm{ADH})$ and reninangiotensin-aldosterone pathways. What is proven however is that goal directed fluid therapy intra-operatively optimises cardiac output and improves outcome [12]. Use of trans-oesophageal Doppler allows for the measurement of stroke volume and cardiac output. Its use has been validated to give accurate changes in cardiac output as opposed to an absolute true value. Fluid therapy can then be directed at keeping pre-load optimised (and hence Starling's curve within its maximal contractility) to reduce the work of the myocardium. It is now given practice to administer relatively small amounts of colloid frequently whilst being guided by the oesophageal Doppler readings. An important point to note is that post operative hypotension should never be blamed on the epidural anaesthesia; a surgical cause should be excluded in the first instance. Although commonplace, hypotension due to epidurals is caused by the blocking of sympathetic fibres in the epidural space. This causes dilation of peripheral blood vessels and decreased peripheral vascular resistance. If longstanding hypotension is problematic then vasopressors could be a better compensator then large volumes of fluid. Decreasing the epidural rate to allow the patient to feel pain is never a solution but its 
reduction can be considered along with adjunct analgesic agents. Surgical technique is evolving to produce minimal trauma to the patient. Often though, a surgeon's desire for minimising the scar or extraction site can lead to overcomplicating the operation itself. Therefore a trade-off needs to be met with minimally invasive surgery including the total operating time, number of ports, extraction site size, benefit to the patient etc. Specimen extraction should be accomplished by the smallest incision possible such as transverse incision for colonic resections and either a modified pfannenstiel or lower midline for rectal resections. Infra-umbilical incisions are preferable as they cause less post-operative pain.

\section{Key Points for Clinical Practice}

- Tailor the type of anaesthesia to the patient and operation

- Avoid long-acting opiates

- Laparoscopic surgery reduces the overall stress response

- Goal-directed fluid therapy reduces complications

\section{Post-operative Interventions}

The patient must be motivated and pain-free to do the things we ask of them. Here also adequate pain relief is essential and augmented pain relief to an epidural or PCA can be considered. The use of agents such as paracetamol (IV or oral), NSAIDs, and atypical agents such as gabapentin and pregabalin should be considered. There is current work underway on combined oxycodone and naloxone agents (Targinact) and early results are promising with a earlier return to gut function. However no consensus on optimal analgesia along with dosing recommendations has been reached thus far. Clearly an approach tailored to the patient and his/her individual needs would be most appropriate. A well-sited epidural, minimal tissue handling, small surgical scars and no "tubes" to tie the patient down are all beneficial. A meta-analysis by Huges et al in the context of open surgery found no differences between epidural and other analgesic modalities with regard to complications and length of stay [13]. Early mobilisation with the patient sitting out of bed the following post-operative day is desirable. Established care plans with motivated staff that can guide and encourage the patient provide the best environment for recovery. We find the establishment of colorectal nurse specialists who visit the patient daily and monitor progress invaluable.

Early feeding is a cornerstone of ERAS and discontinuation of IV fluids should be done as soon as feasible. The prolonged usage of IV antibiotics necessitating peripheral access is no longer the routine. Commencement of carbohydrate drinks should supplement normal dietary intake. The patient should be monitored for early post operative complications and rapid intervention offered.

Patients should be monitored for the development of an ileus, and if identified oral feeding should be curtailed and supportive measures put in place such as IV fluid replacement with high concentrations of electrolytes along with nasogastric drainage. Discharge planning should commence as early as possible. After discharge telephone follow-up at 24 hours is arranged with the Nurse Specialist. This gives confidence to the patients that they are still under an umbrella of care. Patients also have ease of access to the surgical team via the ERAS Nurse Specialist and can have close follow-up and rapid assessment if not progressing along the expected path.

\section{Special considerations}

Many surgeons apply ERAS selectively and proportionally. Whilst this is not incorrect, we feel a proportionate response is merited as one can sometimes predict which of our patients will tolerate the protocol and which will not. This comes with experience but we feel that elements of ERAS can be beneficial to all patient groups.

Application of ERAS in the elderly has been hitherto thought of as unwise. Bagnall et al. [14] performed a systematic review which included the findings of 16 studies. They found ERAS to be safe in the elderly population (>65) with ERAS promoting a reduction in complications and shortened length of stay.

Many of the RCTs that have looked into the efficacy of ERAS are critical of the readmission rate [15]. However a recent systematic

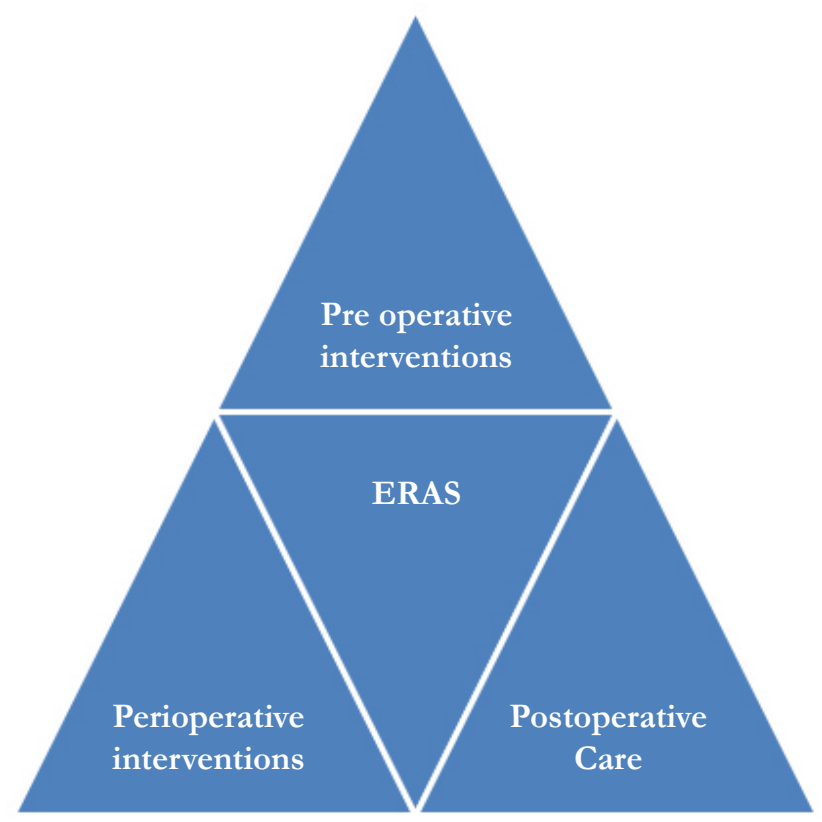


review by Nicholson et al. [16] looked at over 5000 patients. They concluded that there was a reduction in the length of stay, a reduction in the 30-day complication rate but no difference in all cause mortality, major complications or readmission rate. This was also supported by Zhuang et al [17].

Oesophageal Doppler Monitoring is one of six national High Impact Innovations and is eligible for any 2013/14 CQUIN income (Commissioning for Quality and Innovation). This will drive the use of non invasive cardiac output monitoring and stands to deliver significant funding for trusts that are compliant.

Enhanced recovery following colorectal resection has delivered a framework within which patient-centred care can flourish. It divides the patient's journey into stages and ensures the surgical stress inflicted onto the patient is minimised. However one should remember that although all of the interventions are recommendations to the patient, and have been proven to shorten stay, minimise risk and reduce morbidity, a tailored approach with the patient's abilities and wishes should be employed.

\section{Key Points for Clinical Practice}

- Admission on the day of surgery

- Avoidance of bowel preparation

- Avoidance of prolonged fasting

- Carbohydrate loading

- No sedative premedication

- Short-acting anaesthetic agents

- Avoid long-lasting opiates

- Thoracic epidurals or PCA with regional analgesia

- Minimal drains and no nasogastric tubes

- Judicious fluid therapy

- Patient warming

- Thromboprophylaxis

- Minimal "attachments" to the patient

- Early mobilisation

- Prokinetics

- Non-opiate analgesia

- Prevention of nausea and vomiting

- Perioperative oral nutrition

- Audit of compliance

\section{References}

[1]. Ibrahim M, Alazzawi S, Nizam I, Haddad FS (2013) An evidence-based review of enhanced recovery interventions in knee replacement surgery. Ann R Coll Surg Engl 95(6): 386-389.
[2]. Nygren J, Hausel J, Kehlet H, Revhaug A, Lassen K, et al. (2005) A comparison in five European Centres of case mix, clinical management and outcomes following either conventional or fast-track perioperative care in colorectal surgery. Clin Nutr 24(3): 455-461.

[3]. Fearon KC, Ljungqvist O, Von Meyenfeldt M, Revhaug A, Dejong CH, et al. (2005) Enhanced recovery after surgery: a consensus review of clinical care for patients undergoing colonic resection. Clin Nutr 24(3): 466-477.

[4]. Basse L, Hjort Jakobsen D, Billesbolle P, Werner M, Kehlet H (2000) A clinical pathway to accelerate recovery after colonic resection. Ann Surg 232(1): 51-57.

[5]. Svanfeldt M, Thorell A, Hausel J, Soop M, Rooyackers O, et al. (2007) Randomised clinical trial of the effect of preoperative oral carbohydrate treatment on postoperative whole-body protein and glucose kinetics. Br J Surg 94(11): 1342-1350.

[6]. Guenaga KF, Matos D, Castro AA, Atallah AN, Wille-Jørgensen P (2005) Mechanical bowel preparation for elective colorectal surgery. Cochrane $\mathrm{Da}-$ tabase Syst Rev (1): CD001544.

[7]. Veenhof AA, Vlug MS, van der Pas MH, Sietses C, van der Peet DL, et al. (2012) Surgical stress response and postoperative immune function after laparoscopy or open surgery with fast track or standard perioperative care: a randomised trial. Ann Surg 255(2): 216-221.

[8]. Hübner M, Blanc C, Roulin D, Winiker M, Gander S, et al. (2015) Randomized Clinical Trial on Epidural Versus Patient-Controlled Analgesia for Laparoscopic Colorectal Surgery Within an Enhanced Recovery Pathway. Ann Surg 261(4): 648-653.

[9]. Jesus EC, Karliczek A, Matos D, Castro AA, Atallah AN (2004) Prophylactic anastomotic drainage for colorectal surgery. Cochrane Database Syst Rev (4): CD002100.

[10]. Lobo DN, Bostock KA, Neal KR, et al. (2002) Effect of salt and water balance on recovery of gastrointestinal function after elective colonic resection: a randomised controlled trial. Lancet 359(9320): 1812-1818.

[11]. Brandstrup B, Tonnesen H, Beier-Holgersen R, Hjortsø E, Ørding H, et al. (2003) Effects of intravenous fluid restriction on postoperative complications: comparison of two perioperative fluid regimens: a randomised assessor-blinded multicenter trial. Ann Surg 238(5): 641-648.

[12]. Walsh SR, Tang T, Bass S, Gaunt ME (2008) Doppler-guided intra-operative fluid management during major abdominal surgery: systematic review and meta-analysis. Int J Clin Pract 62(3): 466-470.

[13]. Hughes MJ, Ventham NT, McNally S, Harrison E, Wigmore S (2014) Analgesia After Open Abdominal Surgery in the Setting of Enhanced Recovery Surgery: A Systematic Review and Meta-analysis. JAMA Surg 149(12): 1224-1230.

[14]. Bagnall NM, Malietzis G, Kennedy RH, Athanasiou T, Faiz O, et al. (2014) A systematic review of enhanced recovery care after colorectal surgery in elderly patients. Colorectal Dis 16(12): 947-956.

[15]. Varadhan KK, Neal KR, Dejong CH, Fearon KC, Ljungqvist O, et al. (2010) The enhanced recovery after surgery (ERAS) pathway for patients undergoing major elective open colorectal surgery: A meta-analysis of randomized controlled trials. Clin Nutr 29(4): 434-440.

[16]. Nicholson A, Lowe MC, Parker J, Lewis SR, Alderson P, et al. (2014) Systematic review and meta-analysis of enhanced recovery programmes in surgical patients. Br J Surg 101(3): 172-188.

[17]. Zhuang CL, Ye XZ, Zhang XD, Chen BC, Yu Z (2013) Enhanced recovery after surgery programs versus traditional care for colorectal surgery: a metaanalysis of randomized controlled trials. Dis Colon Rectum 56(5): 667-678. 